Review Article

\title{
Focusing on Sodium Glucose Cotransporter-2 and the Sympathetic Nervous System: Potential Impact in Diabetic Retinopathy
}

\author{
Lakshini Y. Herat, ${ }^{1}$ Vance B. Matthews $\mathbb{D}^{1}{ }^{1}$ P. Elizabeth Rakoczy, ${ }^{2}$ Revathy Carnagarin, ${ }^{3}$ \\ and Markus Schlaich ${ }^{3,4}$ \\ ${ }^{1}$ Dobney Hypertension Centre, School of Biomedical Science, University of Western Australia, Crawley, WA, Australia \\ ${ }^{2}$ Lions Eye Institute, Nedlands, WA, Australia \\ ${ }^{3}$ Dobney Hypertension Centre, School of Medicine, University of Western Australia, Crawley, WA, Australia \\ ${ }^{4}$ Department of Cardiology and Department of Nephrology, Royal Perth Hospital, Perth, WA, Australia
}

Correspondence should be addressed to Vance B. Matthews; vance.matthews@uwa.edu.au

Received 6 April 2018; Accepted 14 June 2018; Published 5 July 2018

Academic Editor: Tomohiko Sasase

Copyright (c) 2018 Lakshini Y. Herat et al. This is an open access article distributed under the Creative Commons Attribution License, which permits unrestricted use, distribution, and reproduction in any medium, provided the original work is properly cited.

\begin{abstract}
The prevalence of diabetes is at pandemic levels in today's society. Microvascular complications in organs including the eye are commonly observed in human diabetic subjects. Diabetic retinopathy (DR) is a prominent microvascular complication observed in many diabetics and is particularly debilitating as it may result in impaired or complete vision loss. In addition, DR is extremely costly for the patient and financially impacts the economy as a range of drug-related therapies and laser treatment may be essential. Prevention of microvascular complications is the major treatment goal of current therapeutic approaches; however, these therapies appear insufficient. Presently, sodium glucose cotransporter-2 (SGLT2) inhibitors may offer a novel therapy beyond simple glucose lowering. Excitingly, the EMPA-REG clinical trial, which focuses on the clinically used SGLT2 inhibitor empagliflozin, has been extremely fruitful and has highlighted beneficial cardiovascular and renal outcomes. The effects of SGLT2 inhibitors on DR are currently a topic of much research as outlined in the current review, but future studies are urgently needed to fully gain mechanistic insights. Here, we summarize current evidence and identify gaps that need to be addressed.
\end{abstract}

\section{Diabetes and Its Complications}

Diabetes mellitus is a chronic metabolic disorder marked by high blood glucose levels resulting from defects in insulin secretion, insulin action, or both. At present, diabetes is at pandemic levels. As per the International Diabetes Federation, in the year 2015, the global diabetic population was $\sim 415$ million. By the year 2040, this number is expected to increase to 642 million. All forms of diabetes can lead to the development of diabetes-specific macrovascular and/or microvascular complications. These macrovascular complications commonly affect the arteries of the heart (coronary artery disease), brain, and lower body whereas the microvascular complications can affect small blood vessels of the heart, kidney, foot, and the eye [1]. In this review, we will focus our attention on the microvascular complication of the eye which is known as diabetic retinopathy (DR).

\section{Diabetic Retinopathy (DR)}

Diabetic retinopathy is recognized as one of the most prevalent complications of both type 1 (T1D) and type 2 diabetes (T2D), and it is a leading cause of vision impairment [2]. Globally, the number of people with DR is estimated to grow from 126.6 million in 2011 to 191.0 million by 
2030, and vision-threatening DR is projected to increase from 37.3 million in 2010 to 56.3 million by 2030 [3].

Retinal vascular abnormalities observed via ophthalmoscopy form the basis of the clinical diagnosis for DR [4]. However, a growing body of evidence supports that DR is both a vascular and a neurodegenerative disease of the retina [5]. Characteristic features of DR include hemorrhages, microaneurysms, and intraretinal microvascular abnormalities (IRMA) such as dilated preexisting capillaries, cotton wool spots, hard exudates (lipid deposits), retinal edema, and neovascularization $[2,6]$.

In general, DR is known to progress from a mild nonproliferative DR (NPDR) to a severe proliferative DR (PDR). The early stage of the disease often does not show recognizable retinal changes, leaving patients unaware of the condition. As retinopathy progresses, patients may invariably experience vision loss [6].

The early retinal change in DR is called nonproliferative or background DR. Nonproliferative DR can be subdivided into mild, moderate, severe, and very severe NPDR, according to the modified Airlie House classification of DR severity scale $[4,6]$. During the NPDR stage, patients usually have no symptoms and have normal vision. Although the presence of microaneurysms is the hallmark of NPDR, histopathological observations of diabetic retinas show the presence of pericyte ghosts, acellular capillaries, and thickening of the capillary basement membrane [7] marking the mild or early NPDR stage. However, microaneurysms are the earliest opthalmoscopically detectable, clinical manifestation of DR. Typically, the pericyte to endothelial cell ratio is $1: 1$. Due to diabetes, pericytes necrotise significantly reducing the number of functional pericytes on capillary walls [7]. This selective loss of pericytes from the retinal capillaries is a characteristic lesion that occurs in the very early stages of DR. It is speculated that the loss of pericytes affects the integrity of the capillaries, leading to the formation of acellular capillaries, microaneurysms, or capillary occlusion [8]. Capillary occlusion is histologically manifested as an increased number of acellular capillaries. These capillaries are mere basement membrane tubes lacking endothelial cells and pericyte nuclei [9]. In addition, the mild NPDR stage can also manifest as hemorrhages and/or hard exudates $[4,6]$. The presence of microaneurysms and retinal hemorrhages along with cotton wool spots or IRMA marks the moderate NPDR stage [4]. Moderate NPDR progresses to severe NPDR and shows microaneurysms with venous beading, hemorrhages, or both [6].

Cotton wool spots, also known as soft exudates, are round or oval spots with feathered edges. They appear white, grayish-white, or pale yellow-white under ophthalmoscopic observation. On the other hand, hard exudates are waxy, white, or yellow in colour with sharp edges. IRMA occur in intraretinal blood vessels between the arterioles and venules, closer to areas with capillary nonperfusion. The IRMA can be tortuous blood vessels formed due to intraretinal neovascularization or dilation of preexisting capillaries [10]. When very severe NPDR approaches, features of mild to moderate NPDR increase by severalfold in all four quadrants of the eye [6]. All microvascular changes of NPDR are limited to the inner retina and do not extend beyond the internal limiting membrane [11].

Proliferative DR occurs once NPDR progresses to its next level, with the growth of new blood vessels from preexisting vessels (neoangiogenesis) on the innermost layer of the retina. These blood vessels develop towards the vitreous body and its cavity. The neovascularization or formation of new blood vessels takes place in addition to the clinical features of NPDR. Extensive lack of oxygen leads to the development of neovascularization which is a random growth pattern of blood vessels. Winding of vessels and other various complicated formations indicates neovascular events. The newly formed preretinal vessels are thin, leaky, and fragile in structure and susceptible to hemorrhages, causing the leakage of blood into the vitreous body. As PDR progresses, fibrovascular scar tissue can form around new vessels. This tissue can be vascular or avascular. This fibrovascular variety can lead to retinal tearing and tractional retinal detachment followed by blindness [11]. The exact chronological sequence of development of the above lesions is still incompletely understood.

\section{Molecular Aspects of the Pathogenesis of DR}

One aspect of the pathogenesis of DR is linked to changes in retinal haemodynamics. The decreased blood flow through the retinal tissue leads to retinal hypoxia and has a significant impact on metabolic activities of the retina. The development of a hypoxic environment in the diabetic retina is evident prior to occurrence of other symptoms observed in diabetic patients [12] and diabetic animals [13] and is linked to capillary occlusion, a well-defined mechanism occurring in early DR [14]. As more capillaries become occluded with time, hypoxia in the surrounding retinal tissue enhances vascular endothelial growth factor (VEGF) expression [15]. This promotes breakdown of the blood-retinal barrier, increases vascular permeability, and stimulates endothelial cell growth and retinal neovascularization in the ischemic retina in PDR [16].

A number of other growth factors have been associated with the development of DR and these include plateletderived growth factors (PDGFs), angiopoietin-1 and angiopoietin-2, basic fibroblast growth factor (bFGF), insulin-like growth factor-1 (IGF-1), stromal-derived factor-1, epidermal growth factor (EGF), transforming growth factor-beta 2 (TGF- $\beta 2$ ), and erythropoietin. In addition to these, VEGF has been demonstrated as the main mediator of ocular neovascularization [16].

\section{Perturbed Glucose Homeostasis and DR}

Prolonged duration of diabetes and poorer glycemic and blood pressure control are strongly associated with DR. The storage of glucose in the retina is minimal compared to its high metabolic activity [17]. Thus, the retina is heavily dependent on the systemic circulation for the delivery of adequate glucose [18]. The endothelial cells of the capillaries of the inner blood-retinal barrier and the choroidal vessels across the retinal pigment epithelium (RPE) of the outer 
blood-retinal barrier are responsible for the transport of glucose across the retina. Excessive transport of glucose across the retina leading to high glucose concentrations within cells of the retina is a common factor leading to the pathogenesis of DR. Increased metabolic dysfunction due to high glucose levels and activation of signalling pathways as mentioned below lead to the development and progression of DR. Of the biochemical pathways involved, four major mechanisms have been found to explain the fate of cells and tissues exposed to hyperglycemia (Table 1). These include (1) activation of protein kinase $\mathrm{C}(\mathrm{PKC}),(2)$ increased glucose flow via the polyol pathway, (3) increased advanced glycation end product (AGE) formation, and (4) increased hexosamine pathway flux [19]. All these pathways ultimately lead to increased oxidative stress, inflammation, and vascular dysfunction and upregulation of a wide range of growth factors and eventually contributes to the pathogenesis of DR.

At present, tight control of blood glucose levels is viewed as the primary means by which to disrupt the abovementioned pathways and slow the progression of retinopathy. However, the precise mechanism by which glucose enters the retina and how hyperglycemia causes retinopathy remains unclear. Therefore, in the past two decades, a major focus has been placed in understanding the role and mechanism of glucose transporters in DR.

\section{Glucose Regulatory Mechanisms in DR}

The entry of glucose into cells is regulated by facilitative glucose transporters (GLUTs) and sodium-dependent glucose cotransporters (SGLTs). In the sodium-dependent glucose transport process, SGLT1 and SGLT2 have been well characterised. In particular, SGLT1 is considered to be predominantly expressed in the brush border membrane of mature enterocytes in the small intestine and plays a major role in the absorption of glucose from the lumen of the intestine [20]. In contrast, SGLT2 is mainly found in the S1 and S2 segments of the renal convoluted proximal tubules and is required for the reabsorption of glucose [21].

The GLUT protein family is responsible for the glucose movement within the body, which occurs primary thought 14 isoforms [22]. Of these, GLUT1 and GLUT3 isoforms are expressed in a variety of tissue and cell types in the eye [23]. In particular, GLUT1 is expressed extensively throughout the eye and retina [24-26], suggesting GLUT1 as the main glucose transporter of the eye. In the retina, GLUT1 is highly expressed in pigment epithelial cells, Muller cells, and vascular endothelial cells forming the blood-retinal barrier $[18,25,27,28]$. In contrast, relatively low levels of GLUT3 expression is seen in retinal inner and outer plexiform layers and pigment epithelium. In fact, it is predominantly expressed by the neuronal cells of the retina $[26,29-32]$. Due to pathological characteristics of DR being associated with the loss of blood-retinal barrier function which leads to retinal microvascular abnormalities, GLUT1 and GLUT3 have been investigated in DR. Currently, the role of GLUT3 in DR is not well established. A recent study showed downregulation of GLUT3 in the retina of streptozotocin (STZ) diabetic rats with DR features and suggested this effect was a compensatory response to glucose during hyperglycemia. Furthermore, Knott et al. [32] reported upregulation of GLUT3 mRNA in cultured human retinal endothelial cells exposed to high glucose levels; however, these findings have been controversial due to the expression of GLUT3 being confined to the neurons of the retina [23]. The controversial nature of GLUT3 in DR and the limited number of studies that have investigated its role clearly show the need to further assess the role of GLUT3 in DR.

Given the entry of glucose into the endothelial cells of the inner blood-retinal barrier occurring primarily through GLUT1, its expression and glucose transport could have a major implication on the pathogenic pathways influencing the development and progression of DR. In retinal endothelial cells obtained from postmortem retinas from individuals with long-standing diabetes with minimal or no clinical features of retinopathy, it has been shown that upregulated GLUT1 is localised in the inner blood-retinal barrier. However, this upregulation of GLUT1 can serve to exacerbate the damage to the retinal microvasculature due to ongoing glucose influx [28]. Hypoxia which is a factor associated with the development of more severe retinal lesions in NPDR has been shown to increase GLUT1 expression in retinal endothelial cells [33]. Upregulation of GLUT1 mRNA has been reported in STZ-induced and galactose-fed diabetic rats with DR lesions [34, 35]. Furthermore, knockdown of GLUT1 by intraocular injections of siRNA after 2-4 weeks of STZinduced diabetes in mice has shown restricted glucose transport by inhibiting GLUT1 expression, which decreased retinal glucose concentrations [36, 37]. In the same mouse model, blockade of GLUT1 reduced early biomarkers of DR such as superoxide radicals, chaperone protein b2 crystallin, and VEGF [36]. Taken together, these studies suggest that upregulation of retinal GLUT1 occurs early in DR and this plays a crucial role in disease progression.

Interestingly and important in the current context, the expression of SGLT1 and SGLT2 (Figure 1) has been reported in the eye and the retina [24, 38, 39]. However, the full spectrum of SGLT expression and its role in the eye is poorly understood. Restricted expression patterns of both SGLT1 and SGLT2 have been reported in the lens [24]. It is suggested that SGLT1 is localised at the basolateral membrane of epithelial cells of the chick RPE [38]. The RPE is responsible for transportation of nutrients, ions, and water from the choroidal circulation to the photoreceptors of the retina. Hence, the RPE is essential for the integrity and survival of the neural retina and consequently visual function [40]. In recent years, alteration of both the structural and secretory functions of RPE has been found in DR [41]. The possible localisation of SGLT1 in the basolateral membrane may cause a transmembrane $\mathrm{Na}+$ gradient to drive glucose into the RPE [42], and glucose would diffuse into the neurosensory retina via the apically located GLUT1. Alterations caused to this circuit by high concentrations of glucose may lead to inducible nitric oxide synthase (iNOS) causing nitric oxide-dependent oxidative damage in RPE cells [43]. Although the precise role of SGLT1 in the retina and retinal disease is not well-known, 
TABLE 1: Proposed mechanisms involved in the pathogenesis of DR. Prolonged exposure to hyperglycemia leads to the activation of a number of interconnecting biochemical pathways that contribute to the pathogenesis of DR.

\begin{tabular}{|c|c|c|}
\hline Pathway & Mechanism & Reference \\
\hline Activation of protein kinase $\mathrm{C}(\mathrm{PKC})$ & $\begin{array}{l}\text { (i) Hyperglycemia increases the synthesis of diacylglycerol (DAG). } \\
\text { (ii) Several PKC isoforms (such as PKC- } \beta, \alpha \text {, and } \delta \text { ) are activated. } \\
\text { (iii) Phosphorylation of substrate proteins mediated via PKC promotes changes in retinal } \\
\text { blood flow, increased vascular permeability, endothelial cell dysfunction, and altered } \\
\text { growth factor signaling. } \\
\text { (iv) Retinal ischemia and neovascularization result. }\end{array}$ & {$[62]$} \\
\hline Polyol pathway & $\begin{array}{l}\text { (i) Metabolizes excess glucose to sorbitol and then fructose during hyperglycemia. } \\
\text { (ii) Intracellular accumulation of sorbitol leads to osmotic stress in retinal cells including } \\
\text { ganglion cells, Muller glia, vascular endothelial cells, and pericytes. } \\
\text { (iii) Pathophysiological consequences include thickening of capillary basement } \\
\text { membrane, pericyte loss, formation of acellular capillaries, microaneurysms, } \\
\text { hemorrhages, glial cell activation, and apoptosis. }\end{array}$ & {$[63]$} \\
\hline $\begin{array}{l}\text { Advanced glycation end product } \\
\text { (AGE) formation }\end{array}$ & $\begin{array}{l}\text { (i) Hyperglycemia increases the formation of AGEs. } \\
\text { (ii) Production of AGEs damages target cells via three mechanisms: } \\
\text { (1) Changed extracellular matrix brings about abnormal interactions with matrix } \\
\text { receptor proteins and surrounding matrix components. } \\
\text { (2) AGE-modified intracellular proteins have altered functions. } \\
\text { (3) AGE-modified plasma proteins bind to the receptor for advanced glycation end } \\
\text { products (RAGE) on endothelial cells, leading to the production of reactive oxygen } \\
\text { species. }\end{array}$ & {$[64]$} \\
\hline Increased hexosamine pathway flux & $\begin{array}{l}\text { (i) During hyperglycemia, } \sim 3 \% \text { of glucose is processed through the hexosamine } \\
\text { pathway. } \\
\text { (ii) Fructose-6-phosphate is converted to glucosamine-6-phosphate. } \\
\text { (iii) Subsequently, glucosamine-6-phosphate is metabolized to UDP-GluNAc (uridine } \\
\text { diphosphate N-acetyl glucosamine). } \\
\text { (iv) UDP-GluNAc attaches to serine and threonine residues of transcription factors. }\end{array}$ & {$[65]$} \\
\hline
\end{tabular}

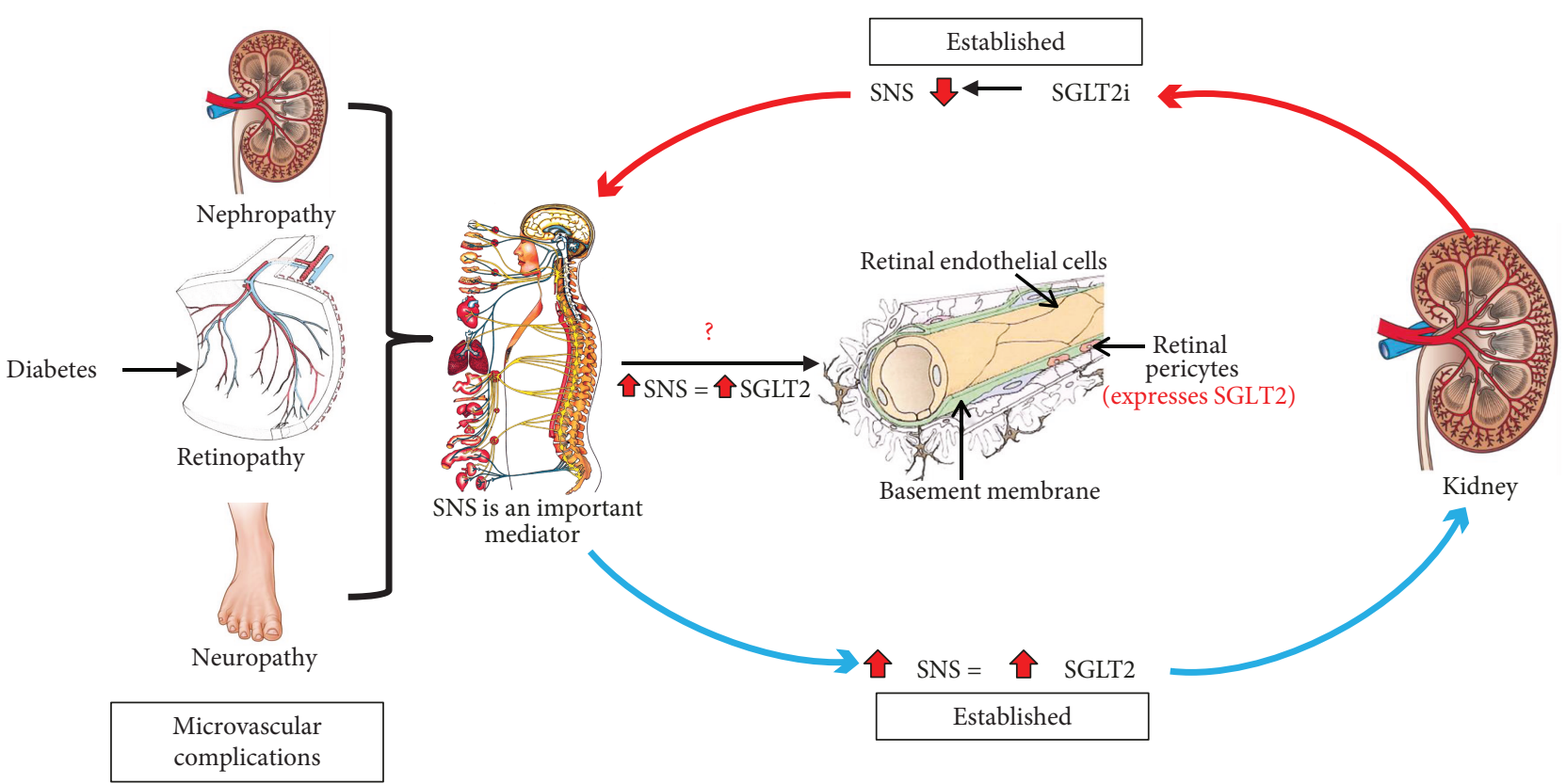

FIGURE 1: The potential molecular role of the SNS and SGLT2 in the pathogenesis of DR. Based on previous studies completed by our team and others, we propose a pivotal role for SGLT2 inhibition (SGLT2i) in the prevention of DR.

the above hypothesis warrants investigations to establish the role of SGLT1 in DR.

Wakisaka and Nagao first reported the expression of SGLT2 in cultured bovine retinal pericytes [39] highlighting the functional glucose sensor role of SGLT2 in the retinal microvasculature. Excessive $\mathrm{Na}+$-dependent glucose entry causes intracellular swelling of pericytes [44] and leads to loss of contractile function, pericyte death, and retinal 
hyperperfusion. Overexpression of the extracellular matrix (such as fibronectin, collagen IV, and laminin) is associated with the development of basement membrane thickening followed by microvascular occlusion and retinal hypoperfusion [45]. This shift in retinal haemodynamics can bring about several downstream triggers of DR. SGLT2 inhibitors have shown to reduce pericyte swelling and overexpression of the extracellular matrix [45]. Arresting the haemodynamic shift early in DR is of significant importance for the development of effective therapeutics for DR.

A new class of antidiabetic drugs act by inhibition of SGLT2, which decreases the reabsorption of glucose from the renal proximal tubules. Hence, specific SGLT2 inhibition (SGLTi) leads to the initiation of glucose excretion via the urine (glucosuria) resulting in reduced blood glucose levels [46]. The SGLT2 inhibitors dapagliflozin, empagliflozin, and canagliflozin have shown to reduce glycosylated haemoglobin levels (HbA1c), fasting plasma glucose levels, blood pressure, and body weight [47-50]. Additionally, it has been shown that SGLT2 inhibitors have protective effects, targeting two of the main complications of diabetes which are cardiovascular disease and kidney-related complications $[47,48]$. These outcomes have been assessed mainly in T2D; however, many studies are currently underway with T1D [51]. Takakura et al. showed for the first time the effect of ipragliflozin on the diabetic retina in spontaneously diabetic Torii fatty rats. In this study, diabetic rats treated with ipragliflozin demonstrated reduced prolongation of oscillatory potential in the electroretinogram, irregularities of the outer nuclear layers of the neural retina were absent, and ipragliflozin inhibited the progression of cataract formation in the lens [52]. The first patient-based study evaluating the impact of dapagliflozin on the retinal microvasculature showed lowered retinal capillary flow and prevented retinal arteriole changes. In this study, the placebo-treated diabetic group showed increased wall-to-lumen ratio indicative of retinal vascular hypertrophy, while the dapagliflozin-treated group showed no changes in wall-to-lumen ratio, indicating the positive effect of dapagliflozin in the prevention of vascular hypertrophy and remodelling [53]. In addition, evidence exists to highlight that dapagliflozin reduced the incidence of DR by $10 \%$ in human T2D cohorts [54]. One possible mechanism of SGLT2i in DR is that it results in blockade of the renin-angiotensin system, improving glycemic control and blood pressure lowering $[55,56]$. A recent metaanalysis conducted by Tang et al. showed that the risk of DR with the treatment of SGLT2i was similar to that with placebo suggesting that SGLT2i did not increase the risk of DR [57]. Thus far, clinical evidence has shown a statistically insignificant beneficial effect. However, none of these studies systematically assessed the retinal vascular and neural pathology in detail during the development and progression of DR. This opens up an opportunity to test the effectiveness of SGLT2 inhibitors in mouse models with retinal and neural damage associated with DR (Figure 1), such as the Akita and Akimba mouse models [5]. The Akita and Akimba mouse models develop diabetes $\sim 4-8$ weeks of age, and these strains progressively develop characteristics of DR ultimately leading to retinal neovascularization, a hallmark feature of
DR. The ultimate goal of effective DR treatments should ideally arrest the progression of the disease to PDR where neovascularization occurs. Therefore, the availability of wellcharacterised DR mouse models such as the Akita and Akimba will provide an ideal vehicle to assess the effectiveness of SGLT2 inhibitors in the pathogenesis and progression of DR. These studies are currently being conducted by our team.

\section{Possible Interaction with the Sympathetic Nervous System}

Recent findings indicate that relevant interactions exist between SGLT2 expression and the sympathetic nervous system. Hyperactivity of the sympathetic nervous system (SNS) is a characteristic feature of obesity and T2D [58, 59]. We have shown for the first time that the major neurotransmitter of the SNS, noradrenaline, upregulates SGLT2 protein expression in human renal proximal tubule cells (Figure 1). In addition, high-fat diet-fed mice treated with dapagliflozin showed a reduction in tissue noradrenaline content and tyrosine hydroxylase expression in both heart and kidney tissue which points to a significant sympathoinhibitory action of SGLT2 inhibitors in our animal model [60]. To date, a limited number of studies have investigated the autonomic nervous system in relation to DR, and these studies suggest DR to be associated with early autonomic dysfunction in T1D and T2D patients [61]. The balance between sympathetic and parasympathetic effects determines the proper function of the autonomic nervous system. We have produced novel data highlighting that in a mouse model of neurogenic hypertension with a substantially activated SNS, neural damage of the outer layer of the retina is evident which is a characteristic feature of DR (Figure 2). We are now conducting mechanistic studies that aim to understand these preliminary findings. As we have already documented [60] that SGLT2 inhibition may downregulate the SNS in the heart and kidney, it is plausible that SGLT2 inhibition may also alleviate detrimental retinal changes that may be underpinned by hyperactivation of the SNS (Figure 1). Our recent results highlight an opportunity to investigate the role of SGLT2 and the SNS in DR.

\section{Alternative Therapies for the Management of Diabetic Retinopathy}

Controlling systemic factors such as blood glucose levels and blood pressure have shown to reduce the development and the progression of nonproliferative to proliferative DR.

Medical interventions such as the use of antiplatelet agents, PKC inhibitors, aldose reductase inhibitors, growth hormone/insulin-like growth factor, and intravitreal administration of corticosteroids and antiangiogenesis agents have proven to be effective at varying levels in limiting visual loss in DR. Among these, antivascular endothelial growth factor (VEGF) therapy administrated into the vitreous cavity represents one of the most beneficial treatment strategies implemented to treat PDR and diabetic macular edema (DME) [16]. 


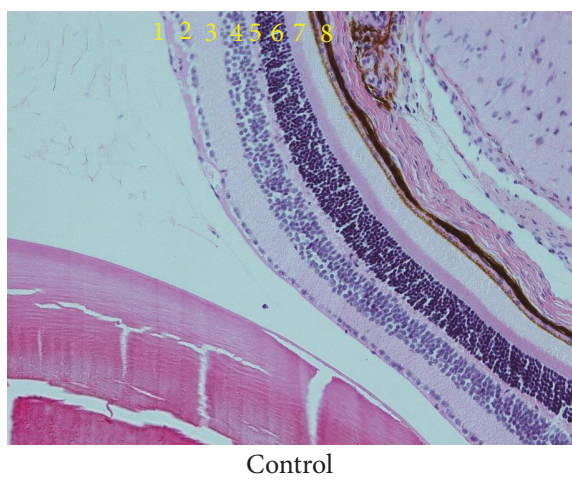

(a)

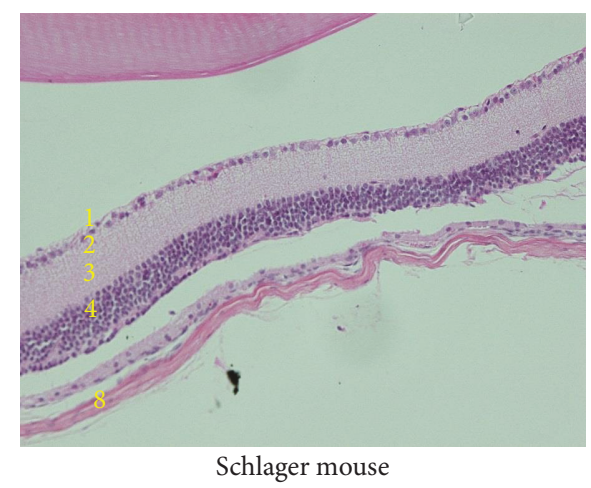

(b)

Figure 2: Sympathetic nervous system hyperactivation in the Schlager mouse is associated with neural damage of the outer layers of the retina. Haematoxylin and eosin staining of the eye of the Schlager mouse highlights that the outer plexiform, outer nuclear, and photoreceptor layers are all absent. 1: nerve fibre layer; 2: ganglion cell layer; 3: inner plexiform layer; 4: inner nuclear layer; 5: outer plexiform layer; 6: outer nuclear layer; 7: photoreceptor layer, and 8: choroid. Magnification: 200x.

Laser and surgical interventions such as panretinal laser photocoagulation and vitrectomy have proven to be effective in treating severe NPDR and PDR. In fact, until recently, panretinal laser photocoagulation was the first and only choice for treating PDR. Furthermore, vitrectomy has been used for the treatment of PDR with vitreous hemorrhages or fibrosis. Vitrectomy is currently used in treating ongoing DME. In the eyes with DME, focal laser treatment has been able to maintain visual acuity without future deterioration. Despite the availability of several treatments, DR remains a major clinical challenge with prevalence expected to rise further over the next decades. Alternative and preventative measures are therefore urgently needed. Based on the limited data available at this stage, therapeutic targeting of the SGLTs, particularly SGLT2, may provide a novel therapeutic opportunity to curb the global burden of DR. Similarly, targeting sympathetic hyperactivity per se and thereby its haemodynamic and inflammatory consequences may provide a useful novel target for DR. Relevant studies are currently being conducted and may well provide further support for such an approach in due course.

\section{Disclosure}

Lakshini Y. Herat and Vance B. Matthews are equal first author.

\section{Conflicts of Interest}

Markus Schlaich has received research support from Boehringer Ingelheim.

\section{Acknowledgments}

The authors acknowledge the generous funding from the Royal Perth Hospital Medical Research Foundation.

\section{References}

[1] M. J. Fowler, "Microvascular and macrovascular complications of diabetes," Clinical Diabetes, vol. 26, no. 2, pp. 77-82, 2008.

[2] D. S. Fong, L. Aiello, T. W. Gardner et al., "Diabetic retinopathy," Diabetes Care, vol. 26, no. 1, pp. 226-229, 2003.

[3] Y. Zheng, M. He, and N. Congdon, "The worldwide epidemic of diabetic retinopathy," Indian Journal of Ophthalmology, vol. 60 , no. 5, pp. 428-431, 2012.

[4] Early treatment diabetic retinopathy study research group, "Grading diabetic retinopathy from stereoscopic color fundus photographs - an extension of the modified Airlie House classification: ETDRS report number 10," Ophthalmology, vol. 98, no. 5, pp. 786-806, 1991.

[5] E. P. Rakoczy, I. S. A. Rahman, N. Binz et al., "Characterization of a mouse model of hyperglycemia and retinal neovascularization," The American Journal of Pathology, vol. 177, no. 5, pp. 2659-2670, 2010.

[6] C. P. Wilkinson, Ferris FL 3rd, R. E. Klein et al., "Proposed international clinical diabetic retinopathy and diabetic macular edema disease severity scales," Ophthalmology, vol. 110, no. 9, pp. 1677-1682, 2003.

[7] M. Mizutani, T. S. Kern, and M. Lorenzi, "Accelerated death of retinal microvascular cells in human and experimental diabetic retinopathy," Journal of Clinical Investigation, vol. 97, no. 12, pp. 2883-2890, 1996.

[8] A. W. Stitt, T. A. Gardiner, and D. B. Archer, "Histological and ultrastructural investigation of retinal microaneurysm development in diabetic patients," British Journal of Ophthalmology, vol. 79, no. 4, pp. 362-367, 1995.

[9] A. J. Barber, D. A. Antonetti, T. S. Kern et al., "The Ins2 ${ }^{\text {Akita }}$ mouse as a model of early retinal complications in diabetes," Investigative Ophthalmology \& Visual Science, vol. 46, no. 6, pp. 2210-2218, 2005.

[10] C. Hudson, "A clinical perspective of diabetic retinopathy," Geriatrics \& Aging, vol. 11, no. 6, pp. 333-341, 2008.

[11] N. C. Steinle and J. Ambati, "Retinal vasculopathies: diabetic retinopathy," in Encyclopedia of the Eye, D. A. Dartt, J. Besharse, and R. Dana, Eds., pp. 109-117, Academic Press, Oxford, 2010. 
[12] A. Harris, O. Arend, R. P. Danis, D. Evans, S. Wolf, and B. J. Martin, "Hyperoxia improves contrast sensitivity in early diabetic retinopathy," British Journal of Ophthalmology, vol. 80, no. 3, pp. 209-213, 1996.

[13] R. A. Linsenmeier, R. D. Braun, M. McRipley et al., "Retinal hypoxia in long-term diabetic cats," Investigative Ophthalmology and Visual Science, vol. 39, no. 9, pp. 16471657, 1998.

[14] A. M. Joussen, T. Murata, A. Tsujikawa, B. Kirchhof, S. E. Bursell, and A. P. Adamis, "Leukocyte-mediated endothelial cell injury and death in the diabetic retina," American Journal of Pathology, vol. 158, no. 1, pp. 147-152, 2001.

[15] L. P. Aiello, J. M. Northrup, B. A. Keyt, H. Takagi, and M. A. Iwamoto, "Hypoxic regulation of vascular endothelial growth factor in retinal cells," Archives of Ophthalmology, vol. 113, no. 12, pp. 1538-1544, 1995.

[16] N. Gupta, S. Mansoor, A. Sharma et al., "Diabetic retinopathy and VEGF," The Open Ophthalmology Journal, vol. 7, no. 1, pp. 4-10, 2013.

[17] C. N. Graymore, "Biochemistry of the retina," in Biochemistry of the eye, C. N. Graymore, Ed., pp. 645-675, Academic Press, 1970.

[18] K. Takata, H. Hirano, and M. Kasahara, "Transport of glucose across the blood-tissue barriers," International Review of Cytology, vol. 172, pp. 1-53, 1997.

[19] M. Brownlee, "The pathobiology of diabetic complications: a unifying mechanism," Diabetes, vol. 54, no. 6, pp. 1615-1625, 2005.

[20] E. M. Wright, "The intestinal Na+/glucose cotransporter," Annual Review of Physiology, vol. 55, no. 1, pp. 575-589, 1993.

[21] Y. Kanai, W. S. Lee, G. You, D. Brown, and M. A. Hediger, "The human kidney low affinity $\mathrm{Na}$ /glucose cotransporter SGLT2. Delineation of the major renal reabsorptive mechanism for D-glucose," The Journal of Clinical Investigation, vol. 93, no. 1, pp. 397-404, 1994.

[22] B. Thorens and M. Mueckler, "Glucose transporters in the 21st century," American Journal of Physiology. Endocrinology and Metabolism, vol. 298, no. 2, pp. E141-E145, 2010.

[23] A. K. Kumagai, "Glucose transport in brain and retina: implications in the management and complications of diabetes," Diabetes/Metabolism Research and Reviews, vol. 15, no. 4, pp. 261-273, 1999.

[24] J. C. Lim, R. D. Perwick, B. Li, and P. J. Donaldson, "Comparison of the expression and spatial localization of glucose transporters in the rat, bovine and human lens," Experimental Eye Research, vol. 161, pp. 193-204, 2017.

[25] A. K. Kumagai, B. J. Glasgow, and W. M. Pardridge, "GLUT1 glucose transporter expression in the diabetic and nondiabetic human eye," Investigative Ophthalmology \& Visual Science, vol. 35, no. 6, pp. 2887-2894, 1994.

[26] G. J. Mantych, G. S. Hageman, and S. U. Devaskar, "Characterization of glucose transporter isoforms in the adult and developing human eye," Endocrinology, vol. 133, no. 2, pp. 600-607, 1993.

[27] K. Takata, T. Kasahara, M. Kasahara, O. Ezaki, and H. Hirano, "Ultracytochemical localization of the erythrocyte/HepG2type glucose transporter (GLUT1) in cells of the bloodretinal barrier in the rat," Investigative Ophthalmology \& Visual Science, vol. 33, no. 2, pp. 377-383, 1992.

[28] A. K. Kumagai, S. A. Vinores, and W. M. Pardridge, "Pathological upregulation of inner blood-retinal barrier Glut1 glucose transporter expression in diabetes mellitus," Brain Research, vol. 706, no. 2, pp. 313-317, 1996.

[29] R. M. Knott and J. V. Forrester, "Role of glucose regulatory mechanisms in diabetic retinopathy," The British Journal of Ophthalmology, vol. 79, no. 11, pp. 1046-1049, 1995.

[30] H. Takagi, H. Tanihara, Y. Seino, and N. Yoshimura, "Characterization of glucose transporter in cultured human retinal pigment epithelial cells: gene expression and effect of growth factors," Investigative Ophthalmology \& Visual Science, vol. 35, no. 1, pp. 170-177, 1994.

[31] T. Watanabe, S. Matsushima, M. Okazaki et al., "Localization and ontogeny of GLUT3 expression in the rat retina," Brain Research. Developmental Brain Research, vol. 94, no. 1, pp. 60-66, 1996.

[32] R. M. Knott, M. Robertson, E. Muckersie, and J. V. Forrester, "Regulation of glucose transporters (GLUT-1 and GLUT-3) in human retinal endothelial cells," The Biochemical Journal, vol. 318, no. 1, pp. 313-317, 1996.

[33] H. Takagi, G. L. King, and L. P. Aiello, "Hypoxia upregulates glucose transport activity through an adenosinemediated increase of GLUT1 expression in retinal capillary endothelial cells," Diabetes, vol. 47, no. 9, pp. 1480-1488, 1998.

[34] S. Roy, A. Kaminski, and J. Zieske, "Distribution and expression of GLUT1 in the retina of galactose-fed rat," Investigative Ophthalmology \& Visual Science, vol. 37, no. 3, pp. S1-S1154, 1996.

[35] R. Poulsom, D. J. Prockop, and R. P. Boot-Handford, "Effects of long-term diabetes and galactosaemia upon lens and retinal mRNA levels in the rat," Experimental Eye Research, vol. 51, no. 1, pp. 27-32, 1990.

[36] L. Lu, C. P. Seidel, T. Iwase et al., "Suppression of GLUT1; a new strategy to prevent diabetic complications," Journal of Cellular Physiology, vol. 228, no. 2, pp. 251-257, 2013.

[37] Z. P. You, Y. L. Zhang, K. Shi et al., "Suppression of diabetic retinopathy with GLUT1 siRNA," Scientific Reports, vol. 7, no. 1, p. 7437, 2017.

[38] L. J. Rizzolo, "Glucose transporters in retinal pigment epithelium development," in Ocular Transporters in Ophthalmic Diseases and Drug Delivery: Ophthalmology Research, J. Tombran-Tink and C. J. Barnstable, Eds., pp. 185-199, Humana Press, Totowa, NJ, USA, 2008.

[39] M. Wakisaka and T. Nagao, "Sodium glucose cotransporter 2 in mesangial cells and retinal pericytes and its implications for diabetic nephropathy and retinopathy," Glycobiology, vol. 27, no. 8, pp. 691-695, 2017.

[40] R. Simó, M. Villarroel, L. Corraliza, C. Hernández, and M. Garcia-Ramírez, "The retinal pigment epithelium: something more than a constituent of the blood-retinal barrierimplications for the pathogenesis of diabetic retinopathy," Journal of Biomedicine \& Biotechnology, vol. 2010, Article ID 190724, 15 pages, 2010.

[41] H. Z. Xu, Z. Song, S. Fu, M. Zhu, and Y. Z. le, "RPE barrier breakdown in diabetic retinopathy: seeing is believing," Journal of Ocular Biology, Diseases, and Informatics, vol. 4, no. 1-2, pp. 83-92, 2011.

[42] J. P. Gnana-Prakasam, R. Veeranan-Karmegam, V. Coothankandaswamy et al., "Loss of Hfe leads to progression of tumor phenotype in primary retinal pigment epithelial cells," Investigative Ophthalmology \& Visual Science, vol. 54, no. 1, pp. 63-71, 2013. 
[43] Z. Yuan, W. Feng, J. Hong, Q. Zheng, J. Shuai, and Y. Gep38MAPK and ERK promote nitric oxide production in cultured human retinal pigmented epithelial cells induced by high concentration glucose," Nitric Oxide, vol. 20, no. 1, pp. 9-15, 2009.

[44] M. Wakisaka, M. Yoshinari, S. Nakamura et al., "Suppression of sodium-dependent glucose uptake by captopril improves high-glucose-induced morphological and functional changes of cultured bovine retinal pericytes," Microvascular Research, vol. 58, no. 3, pp. 215-223, 1999.

[45] S. Roy, E. Bae, S. Amin, and D. Kim, "Extracellular matrix, gap junctions, and retinal vascular homeostasis in diabetic retinopathy," Experimental Eye Research, vol. 133, pp. 58-68, 2015.

[46] A. T. Hattersley and B. Thorens, "Type 2 diabetes, SGLT2 inhibitors, and glucose secretion," The New England Journal of Medicine, vol. 373, no. 10, pp. 974-976, 2015.

[47] B. Zinman, C. Wanner, J. M. Lachin et al., "Empagliflozin, cardiovascular outcomes, and mortality in type 2 diabetes," New England Journal of Medicine, vol. 373, no. 22, pp. 2117-2128, 2015.

[48] C. Wanner, S. E. Inzucchi, J. M. Lachin et al., "Empagliflozin and progression of kidney disease in type 2 diabetes," New England Journal of Medicine, vol. 375, no. 4, pp. 323-334, 2016.

[49] J. Wilding, C. Bailey, U. Rigney, B. Blak, M. Kok, and C. Emmas, "Dapagliflozin therapy for type 2 diabetes in primary care: changes in $\mathrm{HbAlc}$, weight and blood pressure over 2 years follow-up," Primary Care Diabetes, vol. 11, no. 5, pp. 437-444, 2017.

[50] B. Neal, V. Perkovic, K. W. Mahaffey et al., "Canagliflozin and cardiovascular and renal events in type 2 diabetes," The New England Journal of Medicine, vol. 377, no. 7, pp. 644-657, 2017.

[51] A. E. de Leeuw and R. A. de Boer, "Sodium-glucose cotransporter 2 inhibition: cardioprotection by treating diabetes-a translational viewpoint explaining its potential salutary effects," European Heart Journal - Cardiovascular Pharmacotherapy, vol. 2, no. 4, pp. 244-255, 2016.

[52] S. Takakura, T. Toyoshi, Y. Hayashizaki, and T. Takasu, "Effect of ipragliflozin, an SGLT2 inhibitor, on progression of diabetic microvascular complications in spontaneously diabetic Torii fatty rats," Life Sciences, vol. 147, pp. 125-131, 2016.

[53] C. Ott, A. Jumar, K. Striepe et al., "A randomised study of the impact of the SGLT2 inhibitor dapagliflozin on microvascular and macrovascular circulation," Cardiovascular Diabetology, vol. 16, no. 1, p. 26, 2017.

[54] J. Dziuba, P. Alperin, J. Racketa et al., "Modeling effects of SGLT-2 inhibitor dapagliflozin treatment versus standard diabetes therapy on cardiovascular and microvascular outcomes," Diabetes, Obesity and Metabolism, vol. 16, no. 7, pp. 628-635, 2014.

[55] S. J. Shin, S. Chung, S. J. Kim et al., "Effect of sodium-glucose co-transporter 2 inhibitor, dapagliflozin, on renal reninangiotensin system in an animal model of type 2 diabetes," PLoS One, vol. 11, no. 11, article e0165703, 2016.

[56] B. Wang, F. Wang, Y. Zhang et al., "Effects of RAS inhibitors on diabetic retinopathy: a systematic review and meta-analysis," The Lancet Diabetes \& Endocrinology, vol. 3, no. 4, pp. 263-274, 2015.
[57] H. Tang, G. Li, Y. Zhao et al., "Comparisons of diabetic retinopathy events associated with glucose-lowering drugs in patients with type 2 diabetes mellitus: a network metaanalysis," Diabetes, Obesity \& Metabolism, vol. 20, no. 5, pp. 1262-1279, 2018.

[58] N. E. Straznicky, M. T. Grima, N. Eikelis et al., "The effects of weight loss versus weight loss maintenance on sympathetic nervous system activity and metabolic syndrome components," The Journal of Clinical Endocrinology and Metabolism, vol. 96, no. 3, pp. E503-E508, 2011.

[59] A. A. Thorp and M. P. Schlaich, "Relevance of sympathetic nervous system activation in obesity and metabolic syndrome," Journal of Diabetes Research, vol. 2015, Article ID 341583, 11 pages, 2015.

[60] V. B. Matthews, R. H. Elliot, C. Rudnicka, J. Hricova, L. Herat, and M. P. Schlaich, "Role of the sympathetic nervous system in regulation of the sodium glucose cotransporter 2," Journal of Hypertension, vol. 35, no. 10, pp. 2059-2068, 2017.

[61] C. K. Kramer, C. B. Leitão, M. J. Azevedo et al., "Diabetic retinopathy is associated with early autonomic dysfunction assessed by exercise-related heart rate changes," Brazilian Journal of Medical and Biological Research, vol. 41, no. 12, pp. 1110-1115, 2008.

[62] P. Geraldes and G. L. King, "Activation of protein kinase C isoforms and Its impact on diabetic complications," Circulation Research, vol. 106, no. 8, pp. 1319-1331, 2010.

[63] M. Lorenzi, "The polyol pathway as a mechanism for diabetic retinopathy: attractive, elusive, and resilient," Experimental Diabetes Research, vol. 2007, Article ID 61038, 10 pages, 2007.

[64] A. W. Stitt, "AGEs and diabetic retinopathy," Investigative Ophthalmology \& Visual Science, vol. 51, no. 10, pp. 48674874, 2010.

[65] R. A. Kowluru and P. S. Chan, "Oxidative stress and diabetic retinopathy," Experimental Diabetes Research, vol. 2007, Article ID 43603, 12 pages, 2007. 


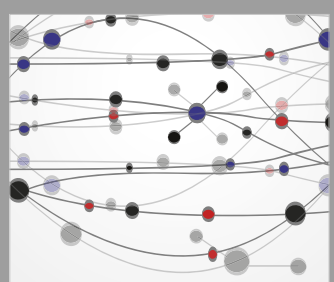

The Scientific World Journal
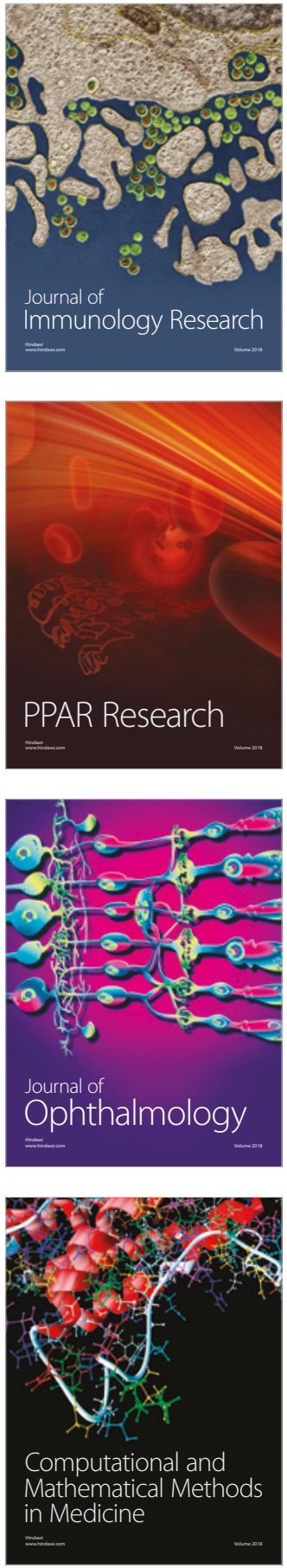

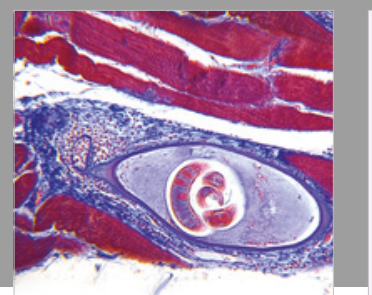

Gastroenterology Research and Practice

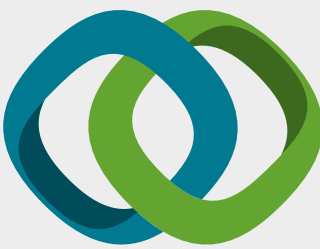

\section{Hindawi}

Submit your manuscripts at

www.hindawi.com
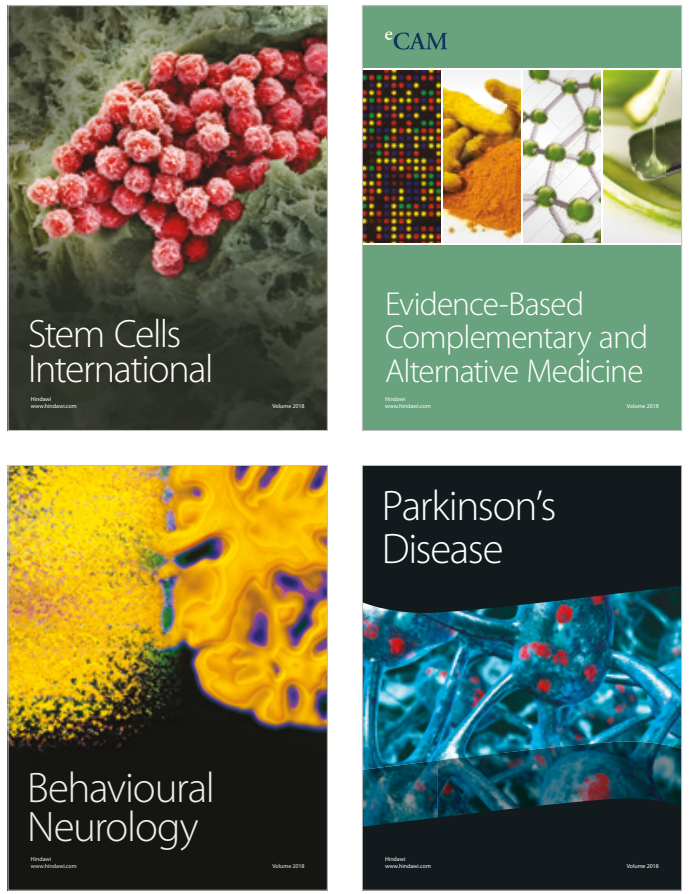

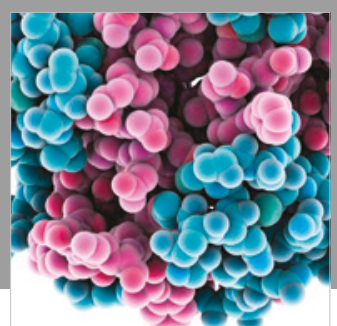

ournal of

Diabetes Research

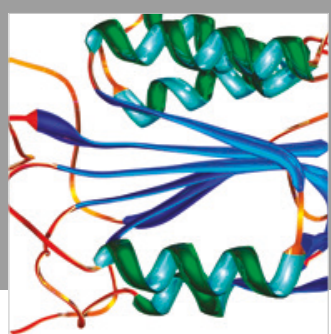

Disease Markers
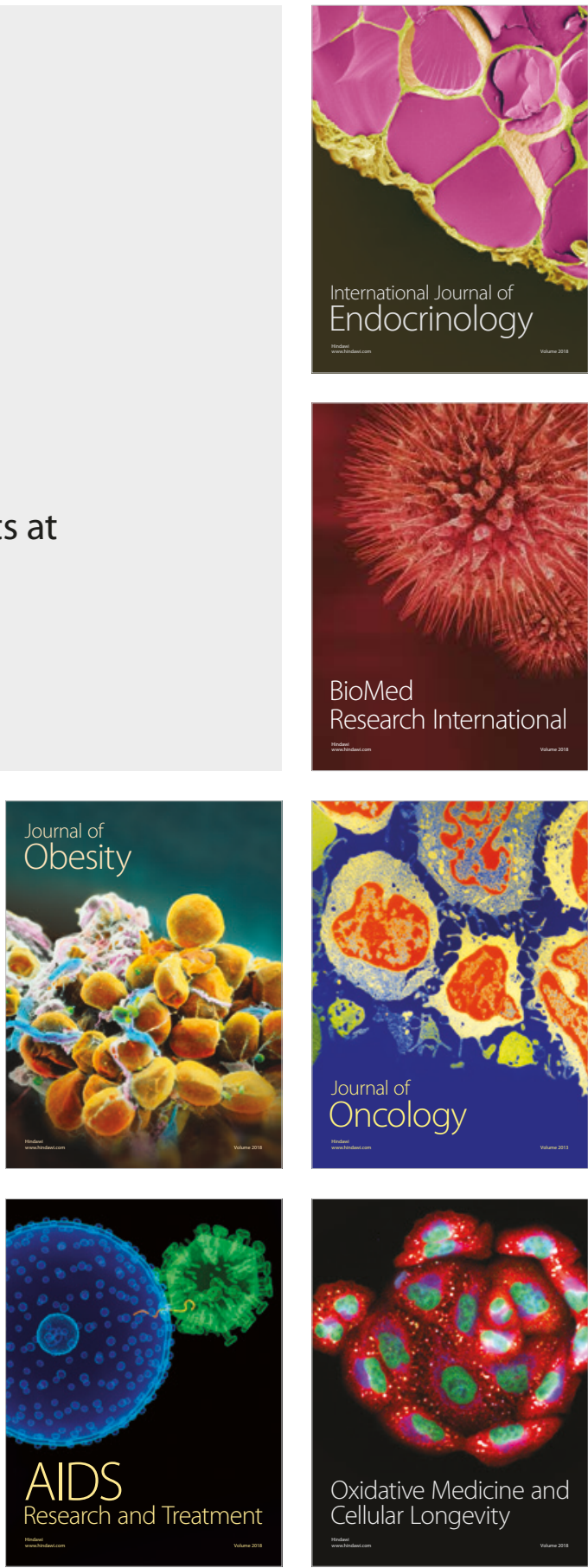